\title{
The efficacy of hemostatic radiotherapy for bladder cancer-related hematuria in patients unfit for surgery
}

\author{
E. Lacarrière, C. Smaali, A. Benyoucef, C. Pfister, P. Grise \\ Department of Urology, Rouen University Hospital (EL, CS, CP, PG) and Department of Radiation Oncology \\ and Medical Physics, Centre Henri Becquerel (AB), Rouen, France
}

\begin{abstract}
Objective: The aim of our study was to assess short and mid-term clinical efficacy of external beam radiation therapy to achieve hemostasis in patients with bladder-cancer related gross hematuria who were unfit for surgery. We also assessed hypofractionation as a possible alternative option for more severe patients.

Patients and Methods: Thirty-two patients were included for hemostatic radiation therapy, with two schedules based on Eastern Cooperative Oncology Group performance status. The standard treatment was $30 \mathrm{~Gy}$ in 10 fractions over 2 weeks. More severe patients underwent a hypofractionated regimen, with 20 Gy in 5 fractions over a one week period. Clinical evaluation was performed at 2 weeks and 6 months.

Results: At 2 weeks, 69\% of patients were hematuria-free. Subgroup analysis showed that $79 \%$ of patients undergoing hypofractionated regimen were hematuria-free. A total of $54 \%$ were hematuria-free with the standard regimen. Based on tumor stage, hematuria was controlled at 2 weeks for $57 \%$ of non-muscle invasive tumors and $72 \%$ of muscle-invasive tumors. After 6 months, 69\% of patients had relapsed, regardless of tumor stage or therapy schedules.

Conclusions: Hemostatic radiotherapy is an effective option for palliative-care hematuria related to bladder cancer in patients unfit for surgery. Although it appears to be rapidly effective, its effect is of limited duration. Hypofractionation also seems to be an effective option; however larger cohorts and prospective trials are needed to evaluate its efficacy compared to standard schedules.
\end{abstract}

\section{ARTICLE INFO}

\section{Key words:}

Hematuria; Urinary Bladder

Neoplasms; Radiotherapy

Int Braz J Urol. 2013; 39: 808-16

Submitted for publication: July 07,2013

Accepted after revision: September 09, 2013

\section{INTRODUCTION}

The incidence of bladder tumors has been reported to be 10.1 per 100000 male patients and 2.5 per 100000 female patients (1). Urothelial carcinomas are primarily found in elderly patients. Two distinct groups of tumors occur: muscle invasive bladder tumors (MIBT) and non-muscle invasive bladder tumors (NMIBT). The management of these patients is very different, and the prognosis involves a minimum amount of risk of local pro- gression and metastasis for more advanced tumors (2). Surgery remains a key element to successful management for both types of tumors.

Elderly patients can become unfit for surgery. These patients must be included in a comprehensive, global care network which attempts to alleviate the patient's symptoms. This remains the main objective of support and palliative care (3).

External beam radiotherapy (EBRT) offers effective tumor control with a minimum amount of invasiveness, and symptom reduction for he- 
morrhage or pain. Unfortunately, the optimal schedule for palliative EBRT in bladder tumors unfit for surgery remains a matter of debate (4).

The aim of our study was to evaluate the efficacy of palliative EBRT for a distressing symptom for patients and their caregivers, i.e. gross hematuria. Two different schedules were used. This provided the more affected population with a hypofractionated regimen, in order to preserve their quality of life.

\section{MATERIALS AND METHODS}

This retrospective, bi-center study was conducted on thirty-two patients treated between January 1993 and January 2009 in the Department of Urology at Rouen University Hospital, Rouen, France and the Becquerel Cancer Center in Rouen, France.

Forty consecutive patients were considered for enrollment. Eight patients were excluded from the study (six prior pelvic radiation therapy, one had Crohn's disease, one had von Willebrand disease). Thirty two patients (Table-1) with bladder cancer were enrolled for palliative pelvic external beam radiation therapy from bladder cancer-related hematuria. All patients were considered unfit for surgical treatment due to age or medical comorbidities. The comorbidities involved are listed in Table- 2 .

Inclusion in this study was based on the following criteria:

Gross hematuria from advanced bladder cancer. All patients were considered grade 3 according to the Common Terminology Criteria for Adverse Events (CTCAE) 4.02 classification.

Histological proof of bladder cancer (NMIBT or MIBT);

1. Failure of local treatment (embolization, electrocoagulation and intravesical instillation of hemostatic agents);

2. Unfit for radical surgical treatment (cystectomy).

Exclusion criteria of this study were:

- Contraindication to perform radiotherapy (i.e. digestive disease);

- Patients submitted to prior radiotherapy in the pelvic area;
- Patients with a coagulation disorder.

All patients were evaluated by an urologist and a radiation oncologist, and had previously undergone transurethral resection of the bladder (TURB). Bladder cancer was confirmed by pathological tests, based on the 2004 WHO classification, and classified into two groups: muscle invasive tumors (MIBT) and non-muscle invasive tumors (NMIBT).

Pathological tumor stage was assessed according to the 2002 modification of the TNM (UICC) classification.

The general health conditions before and after treatment were assessed based on the Eastern Cooperative Oncology Group performance status (ECOG PS Table-3).

External radiotherapy was performed using high energy photon therapy, with four orthogonal beams. The more recent patients underwent a computer tomography (CT) dosimetry scan. The clinical target volume (CTV) was the bladder. Lymph nodes were not considered for treatment, in this palliative setting. Beam definition was performed using a simulator or via a bone localization, with no contrast agent. Additional areas were assessed to take into account bladder filling and patient movements.

Two protocols A and B were used, depending on the general health conditions of the patient as follows: Protocol A (13 patients) delivered 30 Gy in 10 fractions for 2 weeks, if the ECOG PS was less than or equal to 2. Patients in group B (19 patients) underwent a hypofractionated regimen of 20 Gy in 5 fractions for 1 week if they had a ECOG PS of more than 2.

The patients were evaluated 2 weeks and 6 months after the beginning of radiotherapy. The common terminology criteria for adverse events CTCAE version 4.02 (2009 version) (5) published by the US Department of Health and Human Service was used to estimate the intensity of the hematuria (Table-4).

The treatment was considered successful when the hematuria had completely stopped (grade 1). Relapse was defined as the presence of a gross hematuria during the evaluation consultation or the need for other procedures to achieve hemostasis. 
Table 1 - Patients' characteristics.

\begin{tabular}{lcccc}
\hline & Total & Group A & Group B & p value \\
\hline $\mathbf{n}$ & 32 & 13 & 19 & \\
Age (years) & $80.56[65-93]$ & $75.53[65-87]$ & $84[70-93]$ & 0.006 \\
Male & 20 & 8 & 12 & \\
Female & 12 & 5 & 7 & 0.692 \\
ECOG PS & $2.56[1-4]$ & $1.38[1-2]$ & $3.37[3-4]$ & $<0.0001$ \\
\# Comorbidities & $2.72[1-5]$ & $1.92[1-4]$ & $3.35[1-5]$ & 0.092 \\
& & & & \\
Ta-T1 (\%) & $7(21.9)$ & $1(3.1)$ & $6(18.8)$ & 0.114 \\
T2 (\%) & $12(37.6)$ & $6(18.8)$ & $6(18.8)$ & 0.41 \\
T3 (\%) & $6(18.8)$ & $1(3.1)$ & $5(15.7)$ & 0.192 \\
T4 (\%) & $7(21.8)$ & $3(9.4)$ & $4(12.5)$ & 0.893 \\
G2 (\%) & $3(9.4)$ & $0(0)$ & $3(9.4)$ & 0.139 \\
G3 (\%) & $29(90.6)$ & $13(40.6)$ & $16(59.4)$ & 0.139 \\
N+ & 16 & 5 & 11 & 0.288 \\
M+ & 11 & 3 & 8 & 0.201 \\
\hline
\end{tabular}

Table 2 - Major comorbidities encountered during patientenrollment.

\begin{tabular}{lc}
\hline Comorbidities & Times mentioned \\
\hline Chronic bronchitis (COPD) & 23 \\
Ischemic heart disease & 11 \\
Chronic kidney disease & 10 \\
Morbid obesity & 9 \\
Chronic heart failure & 7 \\
Sleep apnea requiring aid & 7 \\
CKD requiring dialysis & 3 \\
Cirrhosis (Child-Pugh A) & 2 \\
Non-bladder metastatic cancer & 1 \\
\hline
\end{tabular}

Blood transfusion, in the absence of gross hematuria, was not an exclusion criteria for our study.

Statistical analysis was performed using XLSTAT V 7.5 software (Addinsoft, 2004). Two-sided p-values were obtained using Mann-Whitney non-parametric bilateral tests. Results were considered significant when the p-value was below 0.05 .

\section{RESULTS}

Thirty-two patients were evaluated. The main results are shown in Table- 5 .

The mean patient age was 81 years (range 65-93). Mean follow-up length was 25 months (range 7-42). 11 patients (35\%) were alive two years after radiation therapy (RT). Mean length before relapse in the entire cohort was 3.6 months (0-42). Mean global survival was 7 months (3-42).

Group A was younger than group B (75.53 vs. 84 years, $p=0.006$ ). The mean performance 
Table 3 - ECOG - PS Eastern Cooperative Oncology Group performance status.

\begin{tabular}{lc}
\hline ECOG & Description \\
\hline 0 & $\begin{array}{c}\text { Fully active, able to carry on all pre-disease } \\
\text { performance without restriction }\end{array}$ \\
1 & $\begin{array}{c}\text { Restricted in physically strenuous activity but } \\
\text { ambulatory and able to carry out work a light se- } \\
\text { dentary nature, e.g., light house work, office work }\end{array}$ \\
2 & $\begin{array}{c}\text { Ambulatory and capable of all self care but } \\
\text { unable to carry out any work activities. Up and } \\
\text { about more than } 50 \% \text { of waking hours }\end{array}$ \\
& $\begin{array}{c}\text { Capable of only limited self care, confined to } \\
\text { bed or chair more than } 50 \% \text { of waking hours }\end{array}$ \\
& Completely disabled. Cannot on self care. \\
& Totally confined to bed or chair
\end{tabular}

status (PS) based on ECOG criteria was 2.56 for the general cohort. Group A had a lower PS than group B (1.38 vs. 3.37; $p<0.0001)$. The mean number of major comorbidities was 2.72 in the general cohort, with less co-morbidities in group A than group B (1.92 vs. 3.26, $\mathrm{p}=0.092)$.

Twenty-five patients had MIBT (78.2\%) and 7 patients had NMIBT (21.8\%). The majority of patients had tumor stage T2 (37.6\%), and the most frequent tumor grade was grade 3 (90.6\%).
No significant differences between treatment groups were found concerning tumor stage or grade.

Malignancy grade 3 was found in 29 patients (90.6\%), 13 group A patients had a grade 3 tumor, while 16 group B patients had a grade 3 tumor $(\mathrm{p}=0.139)$.

Sixteen patients (50\%) had lymph node involvement. More patients in group B than group A had lymph node involvement (LNI) (5 vs. 11, p = 0.288 ). Eleven patients had metastasis at inclusion (34\%), which was more frequent in group B than in group A ( 8 vs. $3, p=0.201$ ).

Thirteen patients (41\%) underwent the standard EBRT regimen based on ECOG PS test results. Nineteen patients (59\%) had more severe health conditions and were selected to undergo a hypofractionated EBRT regimen.

After two weeks of EBRT, twenty-two patients (69\%) presented no hematuria. There appeared to be a better response to EBRT in the muscle invasive group than in the non-muscle invasive group (Figure-1) although no statistical significance was reached $(72 \%$ hematuria-free vs. 57\% $\mathrm{p}=0.469$ ).

In PS-related subgroup analysis (Figure-2), seven group A patients had no hematuria after two weeks of RT (54\%). In group B, fifteen patients (79\%) were hematuria-free after two weeks of hypofractionated RT $(p=0.139)$.

Table 4 - CTCAE - Common Terminology Criteria for Adverse Events grading for hematuria.

\begin{tabular}{|c|c|c|c|c|c|}
\hline & \multicolumn{5}{|c|}{ Grade } \\
\hline & 1 & II & III & IV & V \\
\hline Hematuria & $\begin{array}{l}\text { Asymptomatic; clinical } \\
\text { or diagnostic observa- } \\
\text { tions only; intervention } \\
\text { not indicated }\end{array}$ & $\begin{array}{l}\text { Symptomatic; } \\
\text { urinary catheter or } \\
\text { bladder irrigation } \\
\text { indicated; limiting } \\
\text { instrumental ADL }\end{array}$ & $\begin{array}{l}\text { Gross hematuria; } \\
\text { transfusion, IV } \\
\text { medications or } \\
\text { hospitalization } \\
\text { indicated; elective } \\
\text { endoscopic, radio- } \\
\text { logic or operativa } \\
\text { intervention indi- } \\
\text { cated. limiting self } \\
\text { care ADL }\end{array}$ & $\begin{array}{l}\text { Life-threatening } \\
\text { consequences } \\
\text { urgent radiologic or } \\
\text { operative interven- } \\
\text { tion indicated }\end{array}$ & Death \\
\hline
\end{tabular}

Definition $=\mathrm{A}$ disorder characterized by laboratory test results that indicate blood in the urine 
Table 5 - Results at 2 weeks and 6 months.

\begin{tabular}{lccc}
\hline & $\mathrm{n}$ & Hematuria at 2 weeks $(\%)$ & Hematuria at 6 months $(\%)$ \\
\hline Ta-T1 & 7 & $3(43)$ & $5(71)$ \\
T2-T4 & 25 & $7(28)$ & $17(68)$ \\
p value & & 0.469 & $\mathrm{~ns}$ \\
ECOG PS 0-2 & 13 & $6(46)$ & $10(77)$ \\
ECOG PS 3-4 & 19 & $4(21)$ & $12(63)$ \\
p value & & 0.139 & $\mathrm{~ns}$ \\
TOTAL & 32 & $10(31)$ & $22(69)$ \\
\hline
\end{tabular}

Figure 1 - Results based on tumor stage (initial staging). The rate of relapse is shown in blue, green and red.

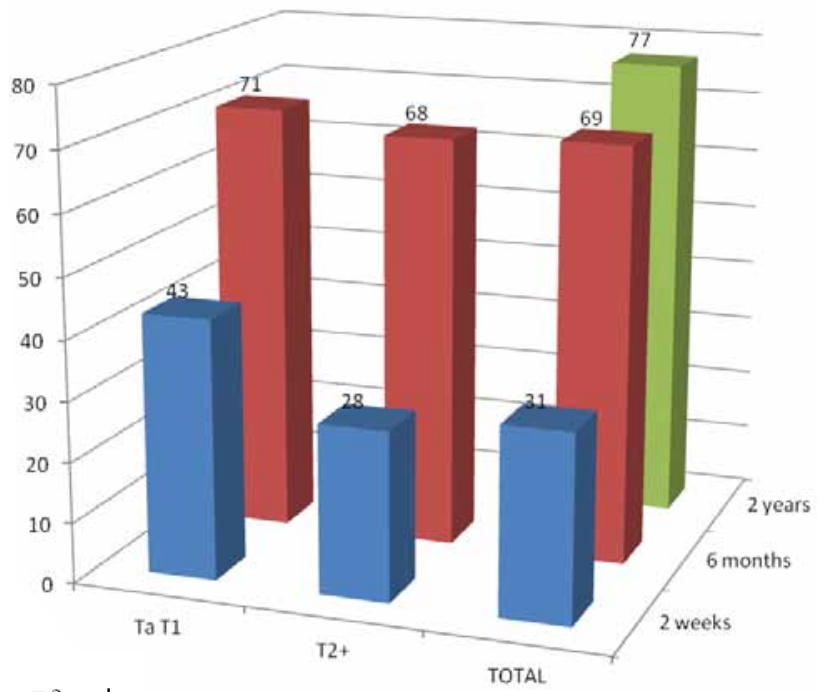

- 2 weeks

- 6 months

- 2 years

After 6 months, both groups showed high relapse rates: 69\% of all patients had relapsed, with no difference in tumor subgroups (62\% vs. $71 \%$ ) or ECOG PS subgroups (77\% vs. 63\%).

There was no statistical difference in the length of the period to relapse found between the subgroups: In tumor stage subgroups (3.1 months vs. $3.3 \mathrm{p}>0.05$ ) or in PS subgroups (3.4 vs. 3.5 months $\mathrm{p}>0.05)$. After 2 years, 7 patients $(23 \%)$ had no hematuria.
Figure 2 - Results based on ECOG - PS Eastern Cooperative Oncology Group performance status (therefore the External beam radiotherapy scheme was used). The rate of relapse is shown in blue, green and red.

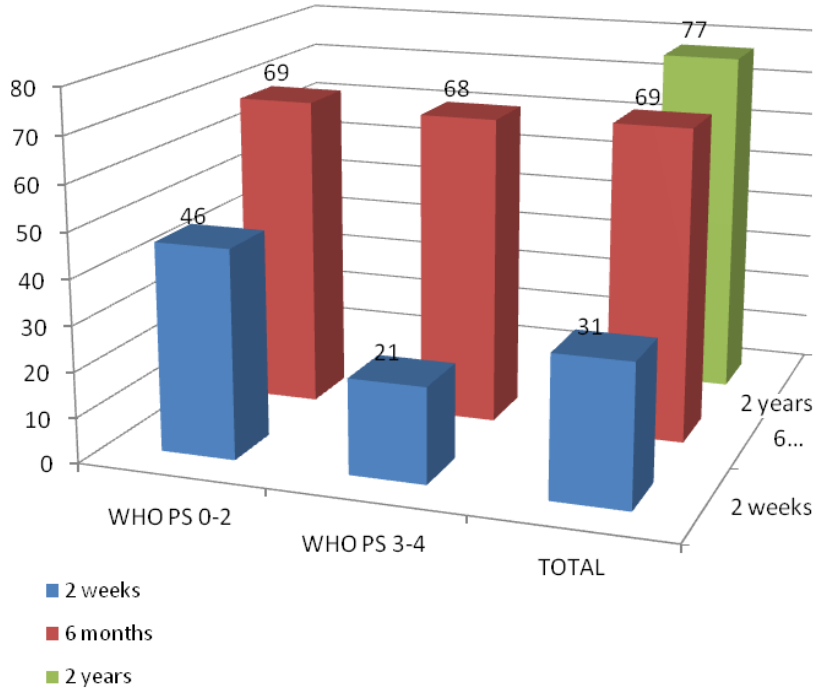

\section{DISCUSSION}

Bladder tumor management remains a routine procedure in clinical urology practice. The standard treatment for non-metastatic bladder tumors remains surgery $(1,3,6)$.

Contra-indication to general anesthesia or epidural anesthesia, due to a severe general state or to severe comorbidities can hinder surgical therapies. For patients with urothelial bladder cancer that are 
unfit for surgery, radiotherapy associated with chemotherapy remains a possible alternative $(3,6,7)$.

However, insufficient local tumor control has been previously reported using this management approach (8). Many patients unfit for surgery will suffer from ongoing tumor-related symptoms (pain, urgency, hematuria). Life expectancy is short (under 9 months) in this population $(2,9)$. Some cases of severe hematuria, especially in patients with cardiovascular history, can be life-threatening (10). Non-surgical hematuria management involves bladder irrigation, coagulation correction, and sometimes intravesical procoagulant agent use or hypogastric arterial embolization. Other treatments have been reported, such as formalin, F2 prostaglandin or alum intravesical instillation, or hyperbaric oxygen therapy. However, results have been disappointing and their use cannot be widely encouraged (11).

Patients with bladder cancer and no surgical option should benefit from support and palliative care, which is defined by the alleviation of symptoms without extending the life expectancy of the patient $(12,13)$. Palliative treatment must strike a balance between efficacy, convenience, toxicity and duration. The main objective of palliative irradiation remains to provide adequate symptomatic relief throughout a patient's anticipated life span while limiting the risk of both acute and late treatment-related complications (14).

Radiation therapy has a double effect on urothelial tumors: Shortly after RT, endothelial cell damage occurs, leading to small and medium vessel rupture or thrombosis. Longer term effects include the disappearance of the microvascular network (15). Other effects that could lead to hemostasis can include: vasoconstriction and platelet aggregation through a decrease in endothelial nitric oxide synthase, an increase in interleukin 1 and TNF alpha through endothelial cell damage, and fibrinolysis inhibition due to the inhibition of plasminogen 1 activator. (16-18).

Hypofractionation involves delivering the same dose as the standard radiation schedule, in a lower number of sessions using a larger daily fraction. This translates into higher long-term tissue toxicity. However, the population submitted to support and palliative care usually has a short life expectancy and bothersome symptoms. Therefore, short-term relief is a more pressing concern than late toxicity. The ease of execution, reduction of patient transportation and rapid efficacy justify the use of hypofractionated radiotherapy for patients in palliative care (19). These facts suggest that more severe patients should be good candidates to hypofractionated EBRT since the impact on their quality of life would be less important.

Therefore, this therapeutic management should be proposed for populations with short-term limited life expectancy. In fact, Scholten et al. reported high rates of 5-year overall complications (33\%) and severe complications (9\%) (20).

According to guidelines, EBRT remains an option to reduce bleeding from various cancers. This palliative and hemostatic approach is mainly used due to its antitumoral effects and easy administration. Moreover, its cytotoxic effects allow for limitation or regression of an irradiated tumor, which can provide a decrease in tumor-specific symptoms $(14,21)$.

The hemostatic effects of EBRT have been previously reported in patients affected by lung carcinomas. Brundage et al. have shown an $80 \%$ reduction in bleeding in non small cell lung cancers. Hypofractionation has also been reported to be as effective as the standard procedure $(22,23)$.

The efficacy of palliative EBRT has also been observed in gynecological cancers. Biswal et al. (24) reported $100 \%$ of bleeding control within three fractions of EBRT. Kraiphibul et al. (25) also observed EBRT efficacy in uterine cervix cancers: $62.9 \%$ of cancer-related bleeding was controlled within three fractions and 97\% within five fractions of radiotherapy.

Duchesne et al. (4) compared two hypofractionated EBRT schedules in a prospective trial, with no difference between a 35 Gy-10 fraction and 21 Gy-3 fraction concerning hematuria improvement (55\% improvement vs. 50\%, $\mathrm{p}=0.198$ ). Clinical evaluation was performed at 3 months. Symptoms such as nocturia, dysuria, and urgency were also improved after EBRT, with no significant difference between the two schedules.

Our retrospective study focused on the hemostatic effect of EBRT on bladder cancer. Other 
urinary symptoms were not taken into account in our data analysis. Limitations include the retrospective nature of this study and the limited patient cohort.

Two EBRT schedules were proposed: 30Gy in 10 fractions (Group A - 2 weeks) or 20Gy in 5 fractions (Group B - 1 week). ECOG PS defined which schedule would be assigned: More severe patients $(E C O G>2)$ underwent the hypofractionated group B procedure. Group B patients were therefore less subjected to transportation, with less impact on their quality of life, and alleged non-inferior efficacy $(4,19,26)$.

As reported in the Duchesne et al. study, long term follow-up in large cohorts was difficult to obtain considering the patient's short-term life expectancy. Therefore, we considered the objective of hemostatic EBRT to be rapidly effective, and well tolerated. Therefore, an early 2 -week medical evaluation was carried-out. Clinical improvement and sustainability was evaluated at the 6-month medical evaluation.

Efficacy following a 2-week evaluation was high: 22 patients (68.75\%) showed no sign of gross hematuria (CTACE grade 1). Rapid control of the bleeding, which was a major objective of the patient care was reported. However, efficacy rates dropped at the 6-month follow-up evaluation: $69 \%$ of the patients had relapsed. This corroborates the findings of Duchesne et al., who reported approximately $60 \%$ of hematuria-free patients 3 months after EBRT with 35 Gy or 21 Gy schedules (4). Two years after EBRT, only 23\% of patients were hematuria-free.

Analysis by ECOG performance status and EBRT schemes suggested a benefit in terms of hematuria control in favor of hypofractionation. The results were once again below the significance threshold: Hypofractionation (Group B) had a $21 \%$ relapse rate after 2 weeks, whereas standard EBRT scheme (Group A) had a 46\% relapse rate (p $=0.139$ ).

The present data suggest that hemostatic EBRT can be an interesting option for patients with hematuria related to bladder cancer unfit for surgery. This therapeutic approach should be used for patients with poor health status, with limited life expectancy, due to the limited sustainability of its effect. Patients with a life expectancy of more than 3 months should not be candidates for hemostatic EBRT. Practitioners should strive to improve the patient's general state of health in order to enable more aggressive, but more effective alternatives.

A benefit was found in hypofractionation in terms of hematuria control, although its use should be considered only for patients with more severe general conditions. These patients were able to benefit most from rapid bleeding control with limited transportation and did not suffer from long-term tissue toxicity due to limited life expectancy. Maintaining quality of life should remain the foremost objective for the management of patients with bladder cancer who are not candidates for surgery.

Srinivasan et al. reported encouraging results with hypofractionated EBRT on advanced bladder cancer (26). Jose et al. reported a positive effect on $62 \%$ of the patients who underwent hypofractionated palliative EBRT, although significant bladder and digestive toxicity was observed, one year after EBRT (27).

Further research is required to establish the higher efficacy of hypofractionated EBRT. Larger cohorts are needed to confirm the trend that we have reported in our retrospective series. This would be useful to define the place of hypofractionated EBRT as the standard therapy for bladder cancer-related hematuria in severe patients unfit for surgical management.

\section{CONCLUSIONS}

Palliative external beam radiation therapy is an effective short-term option for bladder cancer-related gross hematuria when patients are unfit for surgery. Hypofractionation appears to be an interesting option. However, this approach should be used only for patients with poor life expectancy, as its long term toxicity is higher than the standard schedules. Its efficacy compared to usual schedules requires further study.

The effectiveness of hemostatic EBRT seems of limited sustainability, limiting its use to situations when others ways of hemostasis have failed. Hemostatic EBRT cannot take the place of 
surgical options as a means of bleeding control, and it cannot offer long-term control for patients with a longer life expectancy.

\section{ABBREVIATIONS}

$\mathrm{CT}=$ Computer tomography

CTCAE $=$ Common Terminology Criteria for Adverse Events

$\mathrm{CTV}=$ Clinical target volume

ECOG PS = Eastern Cooperative Oncology Group

performance status

$\mathrm{Gy}=$ Grays units of radiation

LNI = Lymph node involvement

MIBT $=$ Muscle invasive bladder tumors

NMIBT $=$ Non-muscle invasive bladder tumors

$\mathrm{RT}=$ Radiation therapy

TURB $=$ Transurethral resection of the bladder

\section{ACKNOWLEDGEMENTS}

The authors are grateful to Richard Medeiros - Medical Editor, Medical Editing International for editing the manuscript.

\section{REFERENCES}

1. Babjuk M, Oosterlinck W, Sylvester R, Kaasinen E, Böhle A, Palou-Redorta J,et al.: EAU guidelines on non-muscle-invasive urothelial carcinoma of the bladder, the 2011 update. Eur Urol. 2011; 59: 997-1008.

2. Duncan W, Quilty PM: The results of a series of 963 patients with transitional cell carcinoma of the urinary bladder primarily treated by radical megavoltage $\mathrm{X}$-ray therapy. Radiother Oncol. 1986; 7: 299-310.

3. EAU Guidelines, edition presented at the 25th EAU Annual Congress. 2010.

4. Duchesne GM, Bolger JJ, Griffiths G0, Trevor Roberts J, Graham JD, Hoskin PJ, et al.: A randomized trial of hypofractionated schedules of palliative radiotherapy in the management of bladder carcinoma: resultsof medical research council trial BA09. Int J Radiat Oncol Biol Phys. 2000; 47: 379-88.

5. Common Terminology Criteria for Adverse Events (CTCAE) Version 4.0. May 28, 2009, U.S Department of Health and Human Services; National Institutes of Health; National Cancer Institute.

6. Pfister C, Roupret M, Wallerand H, Davin JL, Quintens H, Guy L, et al.: Recommendations Onco-Urology 2010: Urothelial tumors. Prog Urol. 2010; 20(Suppl 4): S255-74.
7. Fletcher A, Choudhury A, Alam N: Metastatic bladder cancer: a review of current management. ISRN Urol. 2011; 2011: 545241.

8. Housset M, Maulard C, Chretien Y, Dufour B, Delanian S, Huart $\mathrm{J}$, et al.: Combined radiation and chemotherapy for invasive transitional-cell carcinoma of the bladder: a prospective study. J Clin Oncol. 1993; 11: 2150-7.

9. McLaren DB, Morrey D, Mason MD: Hypofractionated radiotherapy for muscle invasive bladder cancer in the elderly. Radiother Oncol. 1997; 43: 171-4.

10. Pereira J, Phan T: Management of bleeding in patients with advanced cancer. Oncologist. 2004; 9: 561-70.

11. Ghahestani SM, Shakhssalim N: Palliative treatment of intractable hematuria in context of advanced bladder cancer: a systematic review.Urol J. 2009; 6: 149-56.

12. National Institute of Clinical Excellence. Guidance on Cancer Services. Improving Outcomes in Urological Cancers. The Manual. 2002.

13. Brierly $\mathrm{RD}, \mathrm{O}$ 'Brien TS: The importance of palliative care in urology. Urol Int. 2008; 80: 13-8.

14. Hoskin PJ: Radiotherapy in symptom management. Oxford Textbook of Palliative Medicine, 2nd edition, ed. H.G. Doyle D, MacDonald N. 1998: Oxford University Press

15. Fajardo LF: The pathology of ionizing radiation as defined by morphologic patterns. Acta Oncol. 2005; 44: 13-22.

16. Hallahan DE, Spriggs DR, Beckett MA, Kufe DW, Weichselbaum RR: Increased tumor necrosis factor alpha mRNA after cellular exposure to ionizing radiation. Proc Natl Acad Sci U S A. 1989; 86: 10104-7.

17. Woloschak GE, Chang-Liu CM, Jones PS, Jones CA: Modulation of gene expression in Syrian hamster embryo cells following ionizing radiation. Cancer Res. 1990; 50: 339-44.

18. Kanai T, Konno H, Tanaka T, Baba M, Matsumoto K, Nakamura $S$, et al.: Anti-tumor and anti-metastatic effects of humanvascular-endothelial-growth-factor-neutralizing antibody on human colonand gastric carcinoma xenotransplanted orthotopically into nude mice. Int J Cancer. 1998; 77: 933-6.

19. Lutz ST, Chow EL, Hartsell WF, Konski AA: A review of hypofractionated palliative radiotherapy. Cancer. 2007; 109: 1462-70.

20. Scholten AN, Leer JW, Collins CD, Wondergem J, Hermans J, Timothy A: Hypofractionated radiotherapy for invasive bladder cancer. Radiother Oncol. 1997; 43: 163-9.

21. Kynaston HG, Keen CW, Matthews PN: Radiotherapy for palliation of locally advanced prostatic carcinoma. $\mathrm{Br} \mathrm{J}$ Urol. 1990; 66: 515-7.

22. Brundage MD, Bezjak A, Dixon P, Grimard L, Larochelle M, Warde $P$, et al.: The role of palliative thoracic radiotherapy in nonsmall cell lung cancer. Can J Oncol. 1996; 6(Suppl 1): 25-32.

23. Langendijk JA, Aaronson NK, de Jong JM, ten Velde GP, Muller MJ, Slotman BJ, Wouters EF: Quality of life after curative radiotherapy in Stage I non-small-cell lung cancer Int J Radiat Oncol Biol Phys. 2002; 53: 847-53. 
24. Biswal BM, Lal P, Rath GK, Mohanti BK: Hemostatic radiotherapy in carcinoma of the uterine cervix. Int $\mathrm{J}$ Gynaecol Obstet. 1995; 50: 281-5.

25. Kraiphibul P, Srisupundit S, Kiatgumjaikajorn S, Pairachvet $\mathrm{V}$ : The experience in using whole pelvic irradiation in management of massive bleeding from carcinoma of the uterine cervix. J Med Assoc Thai. 1993; 76(Suppl 1): 78-81.

26. Srinivasan V, Brown $\mathrm{CH}$, Turner AG: A comparison of two radiotherapy regimens for the treatment of symptoms from advanced bladder cancer. Clin Oncol (R Coll Radiol). 1994; 6: 11-3.
27. Jose CC, Price A, Norman A, Jay G, Huddart R, Dearnaley DP, et al:: Hypofractionated radiotherapy for patients with carcinoma of the bladder. Clin Oncol (R Coll Radiol). 1999; 11: 330-3.

Correspondence address: Philippe Grise, MD, PhD Department of Urology 1 , rue de Gérmont 76031 Rouen France

Telephone: + 332 3288-8173 E-mail:philippe.grise@chu-rouen.fr 